\title{
A Mathematical Approach Evaluation of Dose Area Product (DAP) Using to Patients Undergoing Intravenous Urography Examinations in Addis Ababa, Ethiopia
}

\author{
Muhdin Abdo ${ }^{1^{*}}$ and Seife Teferi ${ }^{2}$ \\ ${ }^{1}$ Department of Physics, Adama Science and Technology University, Ethiopia \\ ${ }^{2}$ Department of Radiology, School of Medicine, Addis Ababa University, Ethiopia
}

*Corresponding author: Muhdin Abdo, Department of Physics, Adama Science and Technology University, Ethiopia

\begin{abstract}
Patient dose evaluation is one of the quality assurance programs. Dose area product is a product of surface area of patient that exposed to radiation at the skin entrance multiplied by the radiation dose at the surface. This research focused on a dosimetry, DAP, for specific X-rays examination which is known as Intravenous Urography examination. A cross-sectional study was conducted on 145 adult patients age above 16 years examined using IVU in Addis Ababa, Ethiopia at two public and two private hospitals during February 2012-March 2012. The type of X-ray tubes with their outputs (measured using Unfors Ray Safe XI R/F detector) and the exposure parameters for each patients were recorded. The DAP to patients were estimated using mathematical equation. The recorded data was analyzed using statistical software. It was obtained that mean DAP $\left(\mathrm{Gy} \mathrm{cm}^{2}\right)$ per examination within hospitals ranged from 13.7 Gy $\mathrm{cm}^{2}$ to $32.9 \mathrm{~Gy} \mathrm{~cm}^{2}$. The mean value of this study DAP $\left(24.7 \mathrm{~Gy} \mathrm{~cm}^{2}\right)$ per examination which was greater than most of the published results and National diagnostic reference levels. These insure that the Intravenous Urography performed in the some of the hospitals were not capable of acceptable dose levels for patient safety. Therefore, to achieve a lower value of DAP per examination to a patient; a quality assurance program should be done in the hospitals to minimize the number of films per exam and to use appropriate exposure factors to get quality radiographic image for diagnosis.
\end{abstract}

\section{Keywords}

Radiation dose, Dosimetry, Diagnostic reference levels, Quality assurance program

\section{Introduction}

Patient dose evaluation is one of the quality assurance programs (QAP). This research focused on a dosimetry for specific $\mathrm{X}$-rays examination which is known as Intravenous Urography (IVU) examination. IVU does involve a large number of radiographs. IVU is the examination of the urinary tract involving up to 20 radiographs (mean of 8.2) [1-3], 9.3 film on average [4]. For this reason, even if the intravenous urography frequency is only about $1.3 \%$ of the total number of examinations, its contribution to the collective dose is much greater, equal to about $11 \%[5,6]$.

Dose area product (DAP) is a product of surface area of patient that exposed to radiation at the skin entrance multiplied by the radiation dose at the surface. Measurement of dose area product is suitable for achieving optimum degree of safety during radiological examination of patient.

Also, DAP is useful for continuous quality assurance, as well as analysis of performance of X-ray machines. Dose area product could be measured by two methods, namely:

i. Direct measurement through the use of a transmission ionization chamber at the surface of the X-ray tube collimator; and

ii. By mathematical approach (indirect). The mathematical approach involves the product of

\footnotetext{
Citation: Abdo M, Teferi S (2021) A Mathematical Approach Evaluation of Dose Area Product (DAP) Using to Patients Undergoing Intravenous Urography Examinations in Addis Ababa, Ethiopia. Int J Radiol Imaging Technol 7:085. doi.org/10.23937/2572-3235.1510085 Accepted: December 13, 2021; Published: December 15, 2021

Copyright: (c) 2021 Abdo M, et al. This is an open-access article distributed under the terms of the Creative Commons Attribution License, which permits unrestricted use, distribution, and reproduction in any medium, provided the original author and source are credited
} 
irradiated area of the patient and radiation dose incident at the surface.

Many studies have been proposed to measure ESD and DAP in different countries and their results were compared with DRLs recommended by relevant organizations. Also, organizations such as the National Radiological Protection Board (NRPB) and International Atomic Energy Agency (IAEA) recommended the use of dose constraints or investigation levels to provide guidance for medical exposures [7]; IAEA [8]. It was also recommended that dosimetry should be performed regularly to evaluate dose for optimization of radiation protection of the patients in order to introduce Local DRLs [9]. DRLs also help to facilitate standardization and optimization within departments and attempt to reduce dose variations between hospitals.

There is no study on radiation dose level, DAP evaluation in diagnostic radiology in Ethiopia in general, specifically in Addis Ababa, the capital city. Therefore, the objective of the study was to come up with the estimation of Dose Area Product (DAP) for patients undergoing Intravenous Urography examinations using mathematical approach at two private and two public hospitals in Addis Ababa, for potential optimization of radiological doses. Moreover, it is to compare this dose with the international literature.

\section{Methods}

This study utilized a cross-sectional study design. Four X-ray units of the hospitals were included in the study. The hospitals are hereafter referred to as: $\mathrm{H} 1, \mathrm{H} 2, \mathrm{H} 3$ and $\mathrm{H} 4$ hospitals. These hospitals were chosen because they had a considerable number of IVU procedures performed on daily basis. This study was conducted during February 2012 to March 2012 at two government and two private hospitals in the Ethiopian capital Addis Ababa. All governmental/private hospitals were the source population while the study populations were patients who came to take diagnostic IVU examinations in four large private and governmental hospitals.

Self-administered questionnaires regarding each X-ray unit including manufacturer, model, film type and film speed information, socio-demographic characteristics such as age, abdominal thickness of a patient and radiological technical variables (parameters) such as film to focus distance (FFD), film to skin distance (FSD), peak kilo voltage $(\mathrm{kVp})$, tube loading (mAs), current (mA), exposure time (sec), number of exposures to IVU examination were prepared in English and distributed to the radiographers working in the target Hospitals. The completed questionnaires were checked for completeness and consistency and collected from the respective Hospitals.

The annual average number of patients diagnosed in each year using IVU examination before the study period was about 2,160 . The sample size was determined based on Cochran's formula to be 145 patients. Proportional allocation was used to allocate the total sample size to each hospital. Even though, film fault analysis was not performed during the research period; radiographs (films) with acceptable quality and good diagnostic information were selected by the radiographers for this research. This ensured that all dose levels used were representative of diagnostic image.

\section{Dose Area Product (DAP) evaluation}

The DAP is the product of absorbed dose to air multiplied by the irradiated area and is constant at any distance from the focus. The relationship between DAP and ESD for a given field size at FFD is given by the following equation $[4,10]$.

$$
D A P=\frac{E S D}{B S F} *\left(\frac{F S D}{F F D}\right)^{2} * A(F F D)
$$

The Dose Area Product per radiograph (film) was evaluated by using equations (1). For whole examination of a patient was obtained by summing the DAP per exposure of different field size (i.e., area at FFD, $A(F F D))$ of a film sizes $(\mathrm{cm} \times \mathrm{cm})$ used: $30 \times 40 \mathrm{~cm}^{2}$, $24 \times 30 \mathrm{~cm}^{2}$ and $18 \times 24 \mathrm{~cm}^{2}$ used during examination. Entrance Surface Dose (ESD) to a patient including the contribution of the backscatter radiation, BSF [11]. ESD was calculated using the following relation and the published results from [12]:

$$
E S D=(O / P) * m A s *\left(\frac{K v P}{80}\right)^{2} *\left(\frac{100}{F S D}\right)^{2} * B S F
$$

The BSF depends on the X-ray spectrum and beam size. In this study, it was used the BSF equal to 1.39 [4] for all projections, since the BSF variation for the field sizes and kVps used for these examinations is not significant.

The tube output measurement was taken by the principal investigator in a scatter-free geometry, for a peak tube voltage of $80 \mathrm{kVp}$, exposure current-time product of 20 milli-ampere second (mAs) and a focusto-detector distance of $100 \mathrm{~cm}$, using Unfors Ray Safe $\mathrm{XI}$ R/F detector. This dosimeter was calibrated by the manufacturer and reported to have accuracy better than $5 \%$.

The obtained data was recorded and analyzed using statistical software. Before conducting the study, the research project was ethically cleared by the Institutional Review Board of the College of Health Sciences, Addis Ababa University. All participants were informed about the purpose of the study and confidentiality of information. Finally, verbal consent was obtained from each participant.

\section{Results}

The Health institutions $\mathrm{H} 1$ and $\mathrm{H} 2$ (public hospitals) while $\mathrm{H} 3$ and $\mathrm{H} 4$ (private hospitals) all have three phases $X$-ray units. The manufacturer of the machines at $\mathrm{H} 1$ and $\mathrm{H} 3$ was Shimadzu Japan whereas at $\mathrm{H} 2$ is CMET AGCH 


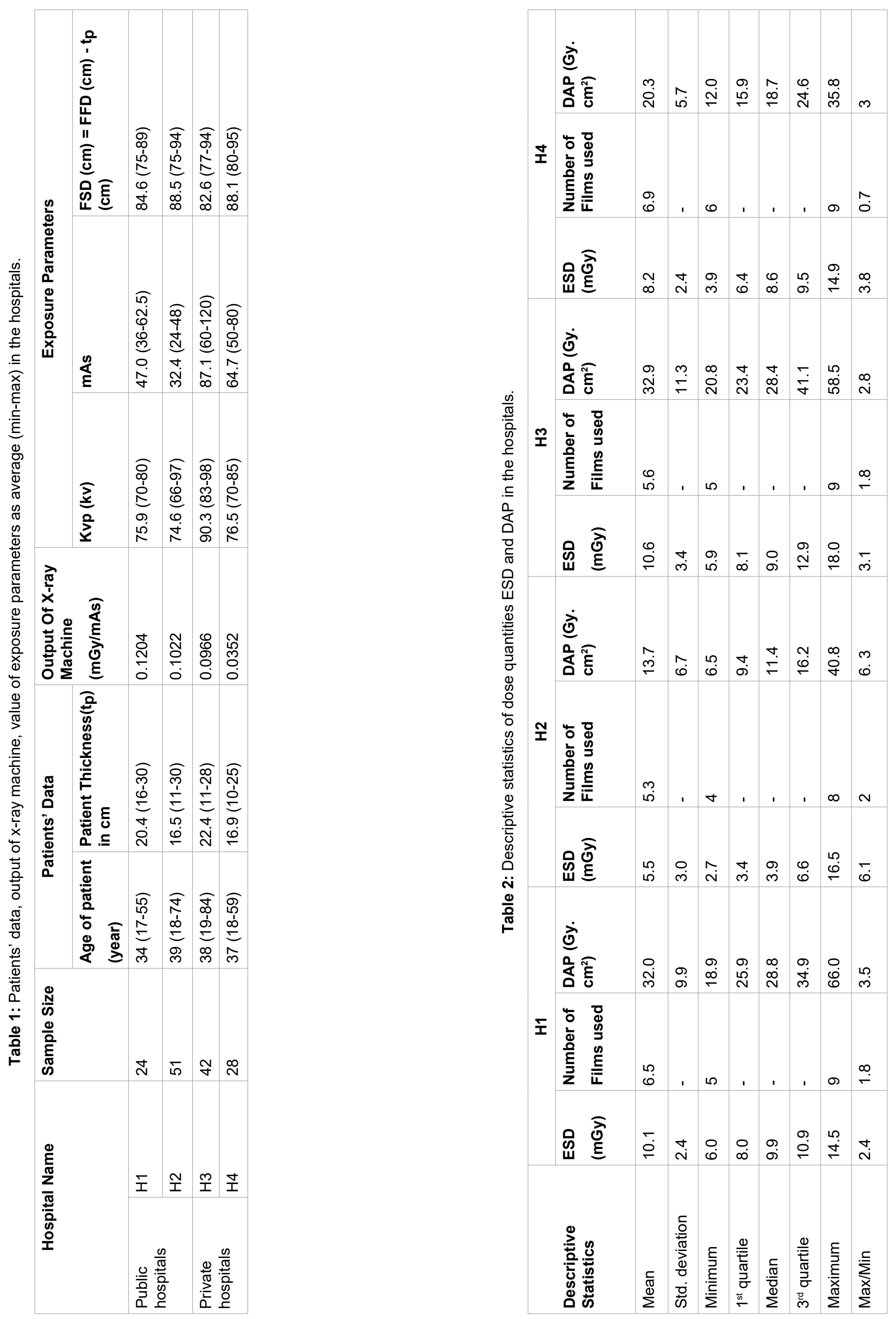




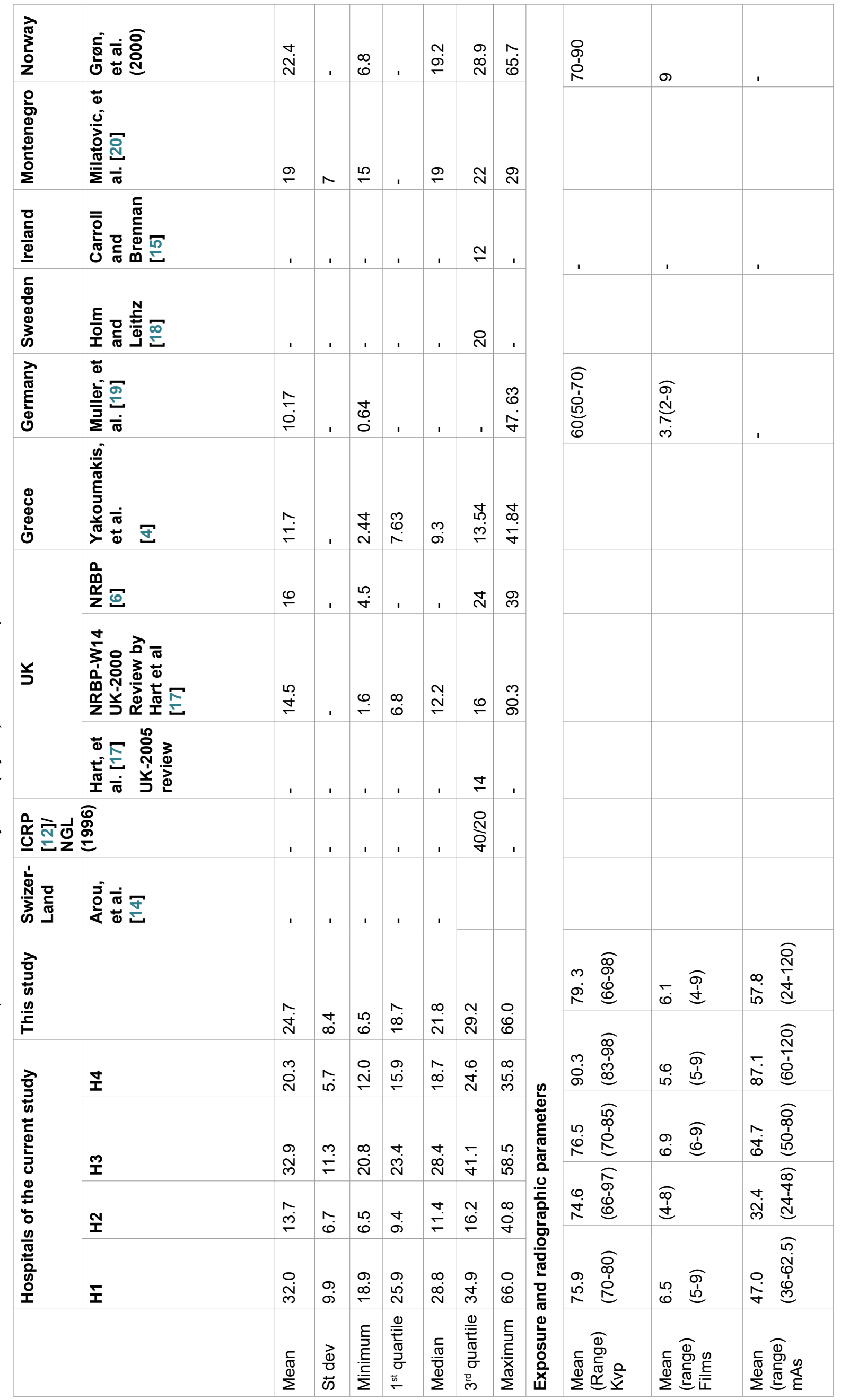


Swizerland, and at $\mathrm{H} 4$ was Villa Viromatic. The output ( $\mathrm{mGy} / \mathrm{mAs}$ ) was determined in free air measurement for a focus-film distance (FFD) of $100 \mathrm{~cm}$ and tube-voltage setting of $80 \mathrm{kv}$ and tube-current of $20 \mathrm{mAs}$.

In this study 145 adults were included. Out of 145 adult patients $70(48 \%)$ adults were from private hospitals while $75(52 \%)$ adults were from public hospitals.

In Table 1, the sample size, the patient data, the output of the $\mathrm{X}$-ray tubes and recorded value of radiographic parameters of the hospitals were shown. The mean (range) age of the patients included in $\mathrm{H} 1$, $\mathrm{H} 2, \mathrm{H} 3$ and $\mathrm{H} 4$ are 34(17-55), 39(18-74), 38(19-84), and $37(18-59)$ respectively. Moreover, for each of the hospitals to the corresponding number of patients the mean (range) values of patient thickness, output of the $\mathrm{X}$-ray machines, and exposure parameters were shown in the table.

In Table 2, descriptive statistics of dose quantities ESD and DAP in Private Hospitals / $\mathrm{H} 3, \mathrm{H} 4 /$ and Public Hospitals /H1, H2/.

In Table 3, descriptive statistics this Study DAP (Gycm2) result in comparison with the results of international studies.

\section{Discussion}

In Table 3, the comparisons of this study results of DAP $\left(\mathrm{Gy} \mathrm{cm}^{2}\right)$ with other international studies and international DRLs were given. In this table, this study mean value of DAP estimated to the patients, $24.7 \mathrm{~Gy}$ $\mathrm{cm}^{2}$ was $62 \%$ and $42 \%$ below the ICRP reference levels of $40 \mathrm{~Gy} \mathrm{~cm}^{2}$ (ICRP, 2001) [13] and the Switzerland DRL of $35 \mathrm{Gycm}^{2}$ [14] respectively. In addition, this study result was $10 \%$ greater than the mean DAP in Norway $[15,16]$ and $3 \%$ greater than UK DRL [17], 23.5\% greater than the Nordic DRL (NGL, 1996), 54\% greater than greater than the Sweden DRL $20 \mathrm{~Gy} \mathrm{~cm}^{2}$ [18], 106\% greater than the Ireland DRL12 $\mathrm{Gy} \mathrm{cm}^{2}$ [15]. These could be due to the set exposure parameters of diagnostically acceptable and longer FFD $>100 \mathrm{~cm}$, a lower number of exposure per examination, utilizing technologically latest type of $x$-ray tube machine, using good type of imaging modality, use of automatic image processor, etc., which are not accessed in developing countries. This study mean DAP to patients was about $52 \%$ greater than the mean DAP of $11.7 \mathrm{~Gy} \mathrm{~cm}^{2}$ in Greece [4]. The mean DAP, $10.17 \mathrm{~Gy}$ $\mathrm{cm}^{2}$ in Germany [19] 59\% greater than this study mean DAP result due to setting a softer tube voltage $150-70$ $\mathrm{kv}$ ) and a lower number of radiograph per examination, 3.7 including the plain abdominal film. The Mean DAP $19 \mathrm{~Gy} \mathrm{~cm}^{2}$ in the developing country Montenegro [20] was $23 \%$ greater than this study mean DAP result, it could be attributed to the use of relatively lower tube loading, lower number 6 (5-7) radiographs per exam, softer mean tube voltage of $67 \mathrm{kv}$ which was a lower mean tube voltage, $79.30 \mathrm{kv}$, and a lower tube output of $0.08 \mathrm{mGy} / \mathrm{mAs}$ than this study.
The mean DAP (24.7 $\left.\mathrm{Gy} \mathrm{cm}^{2}\right)$ per examination and third quartile DAP $\left(29.2 \mathrm{~Gy} \mathrm{~cm}^{2}\right)$ per examination values of this study were greater than most of the published results and national diagnostic reference levels. These insure that the Intravenous Urography performed in the some of the hospitals were not capable of acceptable dose levels for patient safety. Therefore, to achieve a lower value of DAP per examination to a patient; a quality assurance program (QAP) should be done in the hospitals to minimize the number of films per exam and to use appropriate exposure factors for the best of diagnosis of quality radiographic image.

\section{References}

1. Shrimpton PC, Wall BF, Jones DG, Fisher ES, Hillier MC, et al. (1986) A National survey of doses to patients undergoing a selection of routine $\mathrm{X}$-ray examinations in English hospitals. NRPB Report 200. London.

2. Toivonen M, Komppa T (2003) Report on methods of evaluating local skin dose in interventional radiology. Report of the DIMOND III working group of work package 3.1.

3. Toivonen M (2001) Patient dosimetry protocols in digital and interventional radiology. Radiat Prot Dosimetry 94: 105-108.

4. Yakoumakis E, Tsalafoutas A, Nikolaou D, Nazos I, Koulentianos E, et al. (2001) Differences in effective dose estimation from dose-area product and entrance surface dose measurements in intravenous urography. $\mathrm{Br} \mathrm{J}$ Radiol 74: $727-734$

5. (1992) National protocol for patient dose measurements in diagnostic radiology. NRPB, Chilton.

6. (1994) Estimation of effective dose in diagnostic radiology from entrance surface dose and dose-area product measurements. NRPB Report 262.

7. (1990) Patient dose reduction in diagnostic radiology. National Radiological Protection Board (NRPB). Documents for the NRPB, Chilton, Didcot, UK.

8. (1996) International basic safety standards for protection against ionizing radiation and for the safety of radiation sources. International Atomic Energy Agency (IAEA). IAEA safety series, No. 115, Vienna, Austria.

9. Halato MA, Badawi AA, Suliman II, Sulieman AA, Gassom GA, et al. (2010) Radiation doses in intravenous urography and potentials for optimization, tenth radiation physics and protection conference. Nasr City-Cairo, Egypt, 27-30.

10. Toosi MB, Nazery M, Zare H (2006) Application of dosearea product compared with three other dosimetric quantities used to estimate patient effective dose in diagnostic radiology. Iran J Radiat Res 4: 21-27.

11. (2007) Dosimetry in Diagnostic Radiology: An international Code of Practice. Technical reports series; no. 457. IAEA, Vienna.

12. Seife T, Muhdin A, Getachew A (2014) Optimization of radiological doses to patients undergoing Intravenous Urography examinations in Addis Ababa, Ethiopia. Ethiop J Health Dev 28: 202- 210.

13. (2001) International Commission on Radiological Protection (ICRP). Diagnostic reference levels in medical imaging. ICRP Committee 3. 
14. Aroua A, Besancëon A, Buchillier-Decka I, Trueb P, Valley $\mathrm{J}-\mathrm{F}$, et al. (2004) Adult reference levels in diagnostic and interventional radiology for temporary use in Switzerland. Radiat Prot Dosimetry 111: 289-295.

15. Carroll EM, Brennan PC (2003) Investigation into patient doses for intravenous urography and proposed Irish diagnostic reference levels. Eur Radiol 13: 1529-1533.

16. Grøn, P, Olerud HM, Einarsson G, Leitz W, Servomaa A, et al. (1998) Nordic survey of patient doses in diagnostic radiology. Eur Radiol 10: 1988-1992.

17. Hart D, Hillier MC, Wall BF (2002) Doses to patients from medical X-ray examinations in the UK 2000 review. Report NRPB-W14, (Didcot: NRPB)
18. Holm LE, Leitz W (2002) The Swedish Radiation Protection Authority's regulations and general advice on diagnostic standard doses and reference levels within medical X-ray diagnostics. Stockholm, Sweden: Swedish Radiation Protection Authority, SSI FS 2002: 2.

19. Muller M, Heicappelle R, Stainer U, Markele E, Aschoff AJ, et al. (1998) The average dose area product at intravenous urography in 205 adults. Br J Radiol 71: 210-212.

20. Milatovic AO, Ciraj-Bjelac S, Ivanovic $S$, Jovanovic $S$, Spasic-Jokic V (2011) Patient dose measurements in diagnostic radiology Procedures in Montenegro. Radiat Prot Dosimetry 149: 454-463. 